\title{
Germanica
}

\section{Marcel Reich-Ranicki et la Pologne : trajectoire et perceptions}

Audrey KICHELEWSKI: Marcel Reich-Ranicki und Polen: Laufbahn und Wahrnehmungen

Audrey KICHELEWSKI: Marcel Reich-Ranicki and Poland: trajectory and perceptions

\section{Audrey Kichelewski}

\section{OpenEdition \\ Journals}

Édition électronique

URL : https://journals.openedition.org/germanica/7323

DOI : $10.4000 /$ germanica. 7323

ISSN : 2107-0784

\section{Éditeur}

Université de Lille

\section{Édition imprimée}

Date de publication : 1 décembre 2019

Pagination : 17-30

ISBN : 978-2-913857-44-5

ISSN : 0984-2632

Référence électronique

Audrey Kichelewski, « Marcel Reich-Ranicki et la Pologne : trajectoire et perceptions », Germanica [En ligne], 65 | 2019, mis en ligne le 01 janvier 2022, consulté le 08 janvier 2022. URL : http:// journals.openedition.org/germanica/7323; DOI : https://doi.org/10.4000/germanica.7323 


\title{
Marcel Reich-Ranicki et la Pologne : trajectoire et perceptions
}

\author{
Audrey Kichelewski \\ Université de Strasbourg
}

Le 13 juin 2019, le quotidien Frankfurter Allgemeine Zeitung1 consacrait un article à la déferlante de haine antisémite posthume à l'encontre de Marcel Reich-Ranicki, se manifestant dans des commentaires postés sous des vidéos d'anciennes émissions télévisées publiées sur le site internet YouTube ${ }^{2}$, dans lesquelles le critique littéraire, décédé en 2013, donnait des entretiens sur la littérature. Si l'article dénonçait le manque de réactivité de la plateforme de vidéos, peu prompte à supprimer les commentaires calomnieux, il est intéressant de constater qu'au-delà des clichés antisémites habituels déniant au critique sa compétence de jugement sur la littérature allemande du fait d'origines juives perçues par les antisémites comme essentiellement étrangères à la nation allemande, d'autres topoï du discours antisémite sont également déployés, renvoyant quant à eux directement au passé polonais de Reich-Ranicki. Celui-ci est ainsi accusé d'être le « Eichmann de Katowice ». L'allusion renvoie aux quelques semaines que Marcel Reich a passées en janvier 1945 dans cette

1. — Michael Hanfeld, « Youtube lässt Hass gegen Reich-Ranicki zu », Frankfurter Allgemeine Zeitung, 13/06/2019 : https://www.faz.net/aktuell/feuilleton/debatten/antisemitismus-youtube-laesst-hass-gegen-marcel-reich-ranicki-zu-16233418.html (consulté le $9 / 07 / 2019$ ).

2. - Les vidéos ainsi que les commentaires sont toujours visibles, par exemple à l'adresse suivante : https://www.youtube.com/watch?v=Tlr_zPCSCko (consulté le 9/07/2019). 
région tout juste libérée de l'ouest de la Pologne, peuplée d'Allemands et de Volksdeutsche, ces Polonais de langue allemande ayant signé durant la guerre la Volksliste, qui leur octroyait un statut privilégié. La zone s'était couverte de camps de prisonniers où étaient détenus Allemands, Volksdeutsche et bientôt les ennemis du nouveau régime pro-communiste qui se mettait en place. Fugitif du ghetto de Varsovie, caché avec sa femme par un paysan polonais, Marcel venait d'être libéré par l'Armée rouge et s'était porté volontaire dans l'armée polonaise formée en URSS en 1943. Sa connaissance de l'allemand l'avait conduit à être affecté au service de la propagande et de la censure, bientôt rattaché à la police politique, puis à être muté à Katowice. Sa seule présence dans cette région au sortir de la guerre suffit donc aux antisémites de la toile pour dresser un parallèle entre le responsable de l'organisation de l'extermination systématique des Juifs d'Europe et les exactions commises par les autorités communistes, pour lesquelles Marcel Reich-Ranicki travaillait, à l'encontre de la minorité germanophone en Pologne ainsi que des opposants au nouveau régime.

Ce triste constat signale avant tout qu'est sortie de l'ombre et a été portée à la connaissance de l'opinion publique cette partie de la vie de Marcel Reich-Ranicki. Son récit autobiographique $M a$ Vie, publié en $1999^{3}$, laissait déjà une large place à son enfance, dont les premières années passées à Włocławek, au nord-ouest de Varsovie, mais surtout aux années de guerre et d'après-guerre passées en Pologne jusqu'à son départ pour la RFA en 1958. Par la suite, les biographies et articles successifs qui lui furent consacrés en Allemagne comme en Pologne ${ }^{4}$, démontrent tous un vif intérêt pour ces premières années, culminant en 2009 avec le livre de Gerhard Gnauck exclusivement consacré aux « années polonaises » de celui qui fut aussi critique littéraire polonais, ouvrage qui paraît simultanément en allemand et en polonais 5 . Du reste, ce sont les révélations répétées, dans les années 1990, des activités de Marcel Reich-Ranicki durant son séjour dans la Pologne communiste (1945-1958) qui le poussèrent à s'en expliquer, du moins en partie, dans

3. - Marcel Reich-Ranicki, Ma Vie, traduit de l'allemand par Jeanne Etoré, Paris, Grasset, 2001.

4. - Voir notamment Thomas Anz, Marcel Reich-Ranicki, München, Deutscher Taschenbuch Verlag, 2004 ; Uwe Wittstock, Marcel Reich-Ranicki. Geschichte eines Lebens, München, Karl Blessing Verlag, 2005 ; Norbert Honsza, Stephan Wolting (dir.), Marcel Reich-Ranicki. Moja ojczyzna jest literatura [Marcel Reich-Ranicki. Ma patrie est la littérature], Wrocław, Ossolineum, 2007 ; Katarzyna Taborska, Krytyka jako autokreacja. Wizerunki Marcela Reicha-Ranickiego [La critique comme autocréation. Facettes de Marcel Reich-Ranicki], Poznań, Wydawnictwo Poznańskie, 2008.

5. - Gerhard Gnauck, Wolke und Weide. Marcel Reich-Ranickis polnische Jahre, Stuttgart, Klett-Cotta Verlag, 2009. Version polonaise : Marcel Reich-Ranicki. Polskie lata, Varsovie, W.A.B, 2009. 
son autobiographie ${ }^{6}$. Avant de revenir sur le rôle joué par ces révélations dans la manière dont le critique littéraire fut perçu en Pologne, il convient de rappeler que les liens de ce dernier avec ce pays sont profonds et anciens. À l'appui de son autobiographie et de documents d'archives polonais, nous analyserons comment il évoque son pays de naissance et en quoi son témoignage illustre une relation ambivalente tout en enrichissant notre connaissance sur la période de la guerre et de l'après-guerre qu'il a vécues en jeune homme atypique, d'origine juive et de culture allemande.

\section{Une petite enfance polonaise}

Marcel Reich voit le jour le 2 juin 1920 à Włocławek, sur les bords de la Vistule, $150 \mathrm{~km}$ au nord-ouest de Varsovie, dans l'une de ces régions de Pologne marquées, avant la création d'un État polonais, par le mélange des populations, et où l'allemand servait de langue de communication du fait de la proximité avec la Prusse. Ses parents étaient des Juifs assimilés, de culture allemande. Si son père, David Reich, né à Płock, était un commerçant prospère qui parlait russe, yiddish et polonais en plus de l'allemand, demeuré respectueux des traditions religieuses juives, ce n'était pas le cas de sa mère, Hélène Auerbach, fille de rabbin qui avait grandi pauvrement en Allemagne et avait tourné le dos à ses origines pour embrasser pleinement la voie de l'assimilation culturelle. C'est elle qui inscrit le jeune Marcel à l'école allemande, protestante donc, et non à l'école polonaise ou juive, possibilité offerte dans cette ville de 60000 habitants comptant environ un quart de Juifs. Ainsi, le jeune Marcel grandit dans la langue allemande, ce qui lui sera très utile lors de l'émigration de sa famille à Berlin en 1929. C'est la crise économique qui les pousse à chercher un avenir meilleur à Berlin, où vit un oncle avocat chez lequel Marcel est le premier à s'installer, bientôt suivi de ses parents. L'intégration se fera sans peine pour cette famille de culture allemande, à la différence de nombre de leur coreligionnaires polonais ayant fait le même choix, qui restent à l'écart et sont généralement ostracisés par les Juifs allemands ${ }^{7}$, avant que ne finissent par se nouer des contacts culturels sous l'effet de l'expérience commune d'un antisémitisme croissant ${ }^{8}$.

6. - D'après Katarzyna Taborska, Krytyka jako autokreacja ..., op. cit., p. 51.

7. - Sur l'histoire et la place de ces Juifs de l'Est (Ostjuden), voir notamment Trude Maurer, Ostjuden in Deutschland, 1919-1933, Hamburg, Hans Christians Verlag, 1986.

8. - Shulamit Volkov, «The Dynamics of Dissimilation : Ostjuden and German Jews ", in : Jehuda Reinharz, Walter Schatzberg (dir.), The Jewish Response to German Culture. From the Enlightenment to the Second World War, Hanover (NH), University Press of New England, 1985, p. 195-211 ; Delphine Bechtel, « La réception de la littérature yiddish par les intellectuels juifs allemands : aspects des contacts inter- 
Elle est stoppée net par l'avènement du national-socialisme, dont Marcel Reich-Ranicki montre bien dans son autobiographie les effets progressifs sur ses relations amicales au lycée, même si, à Berlin, perdurent grâce à « l'esprit prussien » une certaine correction morale et une volonté d'équité propres aux valeurs bourgeoises, en particulier dans le corps enseignant. Pour autant, Marcel et sa famille furent frappés par l'expulsion du Reich des Juifs ressortissants polonais - ils sont alors environ 50000 - à la fin octobre 1938, au lendemain des accords de Munich entérinant au plan international l'annexion de l'Autriche depuis mars et celle des Sudètes tchécoslovaques. Le gouvernement polonais, craignant l'afflux de Juifs vivant dans ces territoires annexés, conditionne la validité du passeport polonais à l'apposition d'un timbre spécial avant le 30 octobre 1938. Deux jours avant l'échéance, la Gestapo procède à l'arrestation de près de 18000 Juifs polonais résidant en Allemagne, annule leur permis de séjour et les expulse brutalement vers la frontière polonaise. Marcel et ses parents font partie de ces expulsés9.

\section{Être Juif en Pologne durant la Seconde guerre mondiale}

« Tout m'y était étranger » ${ }^{10}$ dit Marcel du pays où il est né et qu'il est contraint de regagner à l'automne 1938. Installés chez son frère aîné demeuré en Pologne, qui avait ouvert un cabinet dentaire à Varsovie, Marcel et sa famille sont bientôt confrontés à la guerre : réquisitions pour travaux forcés, humiliations subies par les Juifs de la ville, prises d'otages arbitraires en représailles d'actes de résistance, marquage par un brassard orné d'une étoile de David et bientôt obligation de s'installer dans le périmètre de la ville défini par l'occupant allemand, ghetto clôturé en novembre 1940 où sont entassés près de 450000 Juifs de Varsovie et des environs.

Le récit autobiographique de Reich-Ranicki et les derniers travaux des historiens sur le ghetto de Varsovie ${ }^{11}$ concordent en tout point. Le texte de Reich-Ranicki est éclairant en particulier sur le rôle du Conseil des Juifs ou Judenrat, nom donné à l'institution confession-

culturels entre Ostjuden et Westjuden » in : Esther Benbassa (dir.), Transmission et passages en monde juif, Paris, Publisud, 1997, p. 451-469.

9. - Sur cette expulsion, voir Bonnie Mae Harris, « From German Jews to Polish refugees : Germany's Polenaktion and the Zbąszyn deportations of October 1938 », Kwartalnik Historii Żydów, n 230, 2009, p. 175-205; Wojciech Olejniczak, Izabela Skórzyńska, Do zobaczenia za rok w Jerozolimie. Deportacje polskich Żydów w 1938 roku z Niemiec do Zbaszynia / See You next Year in Jerusalem. Deportations of Polish Jews from Germany to Zbąszyń in 1938, Zbąszyń, Fundacja TRES, 2012.

10. - Marcel Reich-Ranicki, Ma Vie..., op. cit., p. 115.

11. - Voir notamment Barbara Engelking, Jacek Leociak, The Warsaw Ghetto: $A$ Guide to the Perished City, traduit du polonais par Emma Harris, New Haven, Yale University Press, 2009. 
nelle chargée désormais par l'occupant d'administrer le quotidien du ghetto. La maîtrise de l'allemand assure à Marcel un emploi de traducteur au sein de ce Conseil et ainsi un modeste moyen de subsistance à la famille. Son témoignage montre bien les dilemmes moraux des membres de ce Conseil. C'est Marcel qui lit la directive allemande du 22 juillet 1942 condamnant à la déportation vers Treblinka les Juifs du ghetto, exceptées - provisoirement - certaines catégories, dont les employés du Judenrat et leur famille ; Marcel s'empresse alors d'épouser sa fiancée Tosia. Mais c'est aussi Marcel qui recopie clandestinement ces directives pour les confier à la résistance juive qui documente et vise à faire connaître le sort du ghetto, espérant une intervention alliée qui ne viendra pas. Une partie de ces documents seront retrouvés après la guerre, dans des bidons de lait enfouis sous terre ${ }^{12}$. Du reste, le président du Conseil des Juifs, Adam Czerniaków, ne supportant pas de prendre cette responsabilité d'envoyer ses coreligionnaires à une mort certaine, préféra le suicide, épisode relaté par Reich-Ranicki en hommage à celui qu'il qualifie de « martyr, [de] héros ». Sans doute parce que lui-même fut confronté à ce dilemme moral, auquel il répondit en s'engageant dans la résistance en parallèle de son activité au sein du Judenrat, Marcel Reich-Ranicki défend ces hommes qui furent après la guerre souvent livrés à la vindicte et accusés d'avoir collaboré à la machinerie nazie $^{13}$. La philosophe Hannah Arendt, dans son ouvrage consacré au procès Eichmann, évoqua alors le rôle néfaste de ces institutions dans le processus d'extermination, suscitant depuis régulièrement polémiques et réactions d'historiens attachés à montrer combien la situation dans laquelle les Judenräte étaient placés était complexe ${ }^{14}$.

La richesse du témoignage de Marcel Reich-Ranicki vient aussi de ce qu'il raconte, en précurseur, les détails de la vie quotidienne dans

12. - Sur l'opération de collecte clandestine de documents opérée par l'historien Emmanuel Ringelblum et son équipe, lire Samuel D. Kassow, Qui écrira notre histoire ? Les archives secrètes du ghetto de Varsovie, traduit de l'anglais par Pierre-Emmanuel Dauzat, Paris, Grasset, 2011.

13. - Sur les membres des Conseils juifs et leur jugement après-guerre, voir Jacek Leociak, «Les dilemmes moraux de la collaboration des conseils juifs avec les autorités allemandes dans les ghettos de l'Europe occupée. La spécificité des Conseils juifs dans le cadre de la collaboration avec les Allemands durant la Seconde Guerre mondiale ", Revue d'Histoire de la Shoah, vol. 185, n 2, 2006, p. 379-395.

14. - Hannah Arendt, Eichmann à Jérusalem. Rapport sur la banalité du mal, traduction revue par Martine Leibovici, Paris, Folio Histoire, 2002 [1963], p. 228-240. Pour les travaux d'historiens consacrés aux Conseils juifs, voir notamment Isaiah Trunk, Judenrat. The Jewish Councils in Eastern Europe under Nazi Occupation, London, The Macmillian Company, 1972 ; Raul Hilberg, «Conscious or Unconscious "Tool" », in : Israël Gutman, Cynthia J. Haft (dir.), Patterns of Jewish Leadership in Nazi Europe 1933-1945. Proceedings of the Third Yad Vashem International Historical Conference, Jerusalem, April 4-7, 1977, Jérusalem, Yad Vashem, 1979. 
le ghetto. C'est là qu'il y rencontre l'amour en la personne de Tosia, sa future femme. Il évoque également la vie culturelle, et notamment musicale, comme échappatoire aux malheurs. Après lui, d'autres ouvrages, témoignages ou analyses, mettront l'accent sur cette force de vie et de résistance restée trop longtemps tue, méconnue ou sous-estimée. Citons par exemple l'ultime livre de l'un des héros emblématiques de l'insurrection du ghetto de Varsovie, Marek Edelman, dont le sous-titre est «Et il y avait de l'amour dans le ghetto ${ }^{15}$, le destin du pianiste du ghetto Władysław Szpilman, immortalisé à l'écran en 2002 par Roman Polanski ou encore la trajectoire de la chanteuse Wiera Gran, retracée dans le roman éponyme d'Agata Tuszyńska ${ }^{16}$. Ainsi, l'accent mis par Reich-Ranicki sur la vie culturelle dans le ghetto ne saurait être compris comme un passage obligé d'une narration à visée téléologique, l'auteur réécrivant sa vie de manière à mettre en évidence le destin inéluctable l'ayant conduit à son métier de critique littéraire. Cette vie culturelle non seulement a existé, mais elle est à présent étudiée et pensée comme faisant partie intégrante de la survie des Juifs dans l'Europe occupée ${ }^{17}$.

Le 30 janvier 1943, quelques jours après la deuxième série de rafles organisées dans le ghetto par l'occupant, au cours de laquelle des combattants juifs opposèrent une résistance armée, prélude au grand soulèvement d'avril, Marcel et son épouse décident de s'échapper du ghetto. Là encore, son témoignage sur sa vie de l'autre côté du mur, dans la zone " aryenne » est très précieux, anticipant les recherches menées sur ce mode de survie qui concerna plusieurs milliers de Juifs, dont beaucoup ne virent pas la fin de la guerre, arrêtés sur dénonciation par les nombreux maîtres-chanteurs («szmalcowniki » en polonais) qui sévissaient à Varsovie : " chantage et fuite, cela se reproduisait sans cesse $»^{18}$, écrit Marcel Reich-Ranicki ${ }^{19}$. Les trois conditions qu'il évoque pour pouvoir survivre hors du ghetto - avoir de l'argent, des amis et « un aspect ou un comportement évitant que les Polonais ne

15. - Marek Edelman, La vie malgré le ghetto. Et il y avait de l'amour dans le ghetto, propos recueillis par Paula Sawicka, traduit du polonais par Malgorzata SmoragGoldberg, Paris, Liana Lévi, coll. « Essais », 2010 [2009].

16. - Agata Tuszyńska, Wiera Gran, l'accusée, traduit du polonais par Isabelle Jannès-Kalinowski, Paris, Grasset, 2011 [2010].

17. - À ce sujet, voir notamment Rebecca Rovit, Alvin Goldfarb (dir.), Theatrical Performance during the Holocaust: Texts, Documents, Memoirs, Baltimore and London, The Johns Hopkins University Press, 1999 ; Shirli Gilbert, Music in the Holocaust: Confronting Life in the Nazi Ghettos and Camps, New York, Clarendon Press of Oxford University Press, 2005.

18. - Marcel Reich-Ranicki, Ma Vie..., op. cit., p. 198.

19. - Jan Grabowski, "Je le connais, c'est un Juif! » : Varsovie 1939-1943, Le chantage contre les Juifs, traduit du polonais par Xavier Chantry, Paris, CalmannLevy, 2008 [2004]. 
soupçonnent qu'on était Juif $»^{20}$ - sont tout à fait réalistes et ont été clairement identifiées par une recherche qui, ces dernières années, s'est intéressée aux modalités de survie des Juifs polonais hors des camps et des ghettos. Cette recherche a conclu à l'extrême précarité de la condition de ces fugitifs et à l'étendue des comportements hostiles adoptés par la société polonaise environnante, ce que confirme en tout point le témoignage de Marcel Reich-Ranicki ${ }^{21}$. Reich-Ranicki croise malgré tout le chemin de celui qui sera son sauveteur, le typographe ivrogne Bolek secondé par son épouse Genia. Le portrait qu'il en fait tranche avec l'image souvent idéalisée du « Juste parmi les nations ", saint et héroïque - il est bien davantage conforme à ce que l'on sait à présent de celles et ceux qui risquèrent leur vie pour aider des Juifs 22 : Bolek est un marginal, critique envers l'Église, un prolétaire, un homme instruit qui vit de petites combines et de trafics. L'aide fut probablement monnayée ${ }^{23}$. Marcel et sa femme fabriquent pour lui la nuit des cigarettes que Bolek revend, et corrigent à sa place les devoirs des enfants des environs, faisant office de professeurs doublement clandestins. Cela n'empêchera pas la famille de recevoir, à titre posthume en 2006, la médaille des Justes parmi les Nations.

Marcel Reich-Ranicki n'a pas attendu d'écrire son autobiographie pour témoigner de son itinéraire durant la Seconde Guerre mondiale, d'autant plus singulier qu'il fait partie des quelques dizaines de milliers de Juifs seulement qui ont pu survivre sur le territoire de la Pologne occupée ${ }^{24}$. En effet, il témoigne une première fois, comme beaucoup de rescapés de la Shoah en Pologne, auprès de la Commission centrale historique juive mise en place dès 1945 pour recueillir la parole des survivants. Il complète ses dires en 1951 pour narrer cette fois son rôle dans le soulèvement armé du ghetto et en particulier son « attaque contre la caisse du Judenrat $»^{25}$. À cette époque, le directeur de l'Institut d'his-

20. - Marcel Reich-Ranicki, Ma Vie..., op. cit., p. 193.

21. - Pour une littérature en français sur ces questions, voir notamment Barbara Engelking, «On ne veut rien vous prendre, seulement la vie ! " des Juifs cachés dans les campagnes polonaises, 1942-1945, Paris, Calmann-Levy, 2015 [2011] et Jan T. Gross, Irena Grudzinska Gross, Moisson d'or : Le pillage des biens juifs, Paris, Calmann-Levy, 2014 [2011].

22. - Nechama Tec a établi une typologie des sauveteurs qui fait ressortir leur marginalité sociale. Voir When Light pierced the darkness : Christian Rescue of Jews in Nazi-Occupied Poland, Oxford, Oxford University Press, 1986.

23. - La fille de Bolek l'affirme dans un entretien accordé à Gerhard Gnauck, Marcel Reich-Ranicki..., op. cit. p. 62-63.

24. - On estime leur nombre à 60000 tout au plus, pour une population juive en Pologne en 1939 de plus de trois millions de personnes. Voir Audrey Kichelewski, Les survivants. Les Juifs en Pologne depuis la Shoah, Paris, Belin, 2018, p. 20-21.

25. - Archives de l'Institut d'histoire juive (AŻIH), volume 301 (témoignages), $\mathrm{n}^{\circ} 400$ (sans date, probablement 1954) et 5010 (daté du 8 octobre 1951). 
toire juive, Bernard Mark, prépare un ouvrage sur la résistance dans le ghetto de Varsovie et recherche des témoignages d'actions menées contre le Conseil juif, qu'il s'agit de dépeindre, conformément à l'idéologie d'alors, comme un organe bourgeois et hostile au prolétariat juif ${ }^{26}$. Mark doit également répondre devant la commission du Parti du rôle de Ranicki dans le Judenrat pendant la guerre, ce qui explique que le témoignage de Reich-Ranicki ait été recueilli à l'Institut d'histoire juive cette même année 1951, dans le contexte de son conflit avec le Parti27. Après son départ de Pologne, Marcel Reich-Ranicki témoigne à nouveau en 1961, cette fois-ci en RFA, dans le cadre de la commission d'enquête sur les crimes nazis, établie à Ludwigsburg en 1958, certainement en vue de la préparation des procès contre les criminels nazis qui se tiendront en RFA dans les années soixante. Enfin, en 2009, il donne un entretien pour un film consacré à la vie culturelle dans le ghetto de Varsovie réalisé par le musée de Yad Vashem à Jérusalem ${ }^{28}$.

\section{Dans la Pologne communiste : " un censeur et un communiste dans la police politique ${ }^{29}$}

Dès leur libération par l'Armée Rouge en septembre 1944, Marcel Reich-Ranicki et sa femme gagnent la ville de Lublin, alors capitale du gouvernement polonais de libération nationale, et se portent volontaires dans l'armée populaire polonaise formée l'année précédente en Union soviétique, afin d'aider à la victoire finale sur le Troisième Reich. De nouveau, c'est la parfaite connaissance de l'allemand bien plus que son état physique déplorable qui permet à Marcel d'être engagé au service de la censure de l'armée et bientôt de sa branche du ministère de Sécurité

26. - Sur Bernard Mark et les conditions d'écriture de l'histoire à l'Institut d'histoire juive dans les années 1950, voir Jean-Charles Szurek, «Être témoin sous le stalinisme. Les premières années de l'Institut Historique Juif de Varsovie », in : Delphine Bechtel, Evelyne Paltagean, Jean-Charles Szurek, Paul Zawadzki (dir.), Écritures de l'histoire et identité juive. L'Europe ashkénaze, $X I X^{e}-X X^{e}$ siècles, Paris, Les Belles Lettres, 2003, p. 51-82.

27. - Voir plus loin sur l'accusation portée contre Marcel d'avoir fait partie du Judenrat. Sur la manière dont Mark prit la défense de Ranicki, voir AAN (Archives des Actes Nouveax), KC PZPR (Comité central du Parti ouvrier unifié polonais, CKKP (Commission centrale de Contrôle du Parti), dossier 2139/55, " Opinion de Bernard Mark », p. 60, cité dans Paweł Libera, « Marcel Reich-Ranicki... », art. cit., p. 256.

28. - Film accessible en ligne sur le site internet du fils de Marcel ReichRanicki, à l'adresse suivante : https://www.maths.ed.ac.uk/ v1ranick/musicinghetto. mp4 (consulté le 12 juillet 2019).

29. - Sous-titre extrait d'un article critique sur Reich-Ranicki, écrit par l'historien polonais Paweł Libera, « Marcel Reich-Ranicki przed Centralną Komisją Kontroli Partynej (1950-1957) » [Marcel Reich-Ranicki devant la Commission centrale de contrôle du Parti], Zeszyty historyczne, t. 546, Maisons-Laffitte, Association Institut Littéraire Kultura, 2009, p. 186. 
publique. C'est essentiellement cette partie-là de sa vie, marquée par son engagement dans le parti communiste en janvier 1945 - indispensable pour occuper les fonctions politiques auxquelles on le destinait - qui a suscité ces dernières années le plus d'études et de commentaires, en Allemagne comme en Pologne. Ceux-ci firent en particulier suite à la parution en 2009 du livre de Gerhard Gnauck déjà évoqué, consacré aux années polonaises de Reich-Ranicki, dans le but explicite de compléter une autobiographie jugée trop « subjective », comme le souligne Norman Davies, l'historien britannique spécialiste de la Pologne chargé de préfacer l'édition polonaise de Gnauck. Dès lors, il s'agit pour beaucoup de rechercher la nature précise des activités de Reich-Ranicki en Pologne, dans les services secrets notamment, afin de mettre au jour des éléments qu'il aurait délibérément occultés dans son autobiographie et cela, dans le but, inavoué, de ternir sa réputation. Si dans son livre Gnauck, journaliste allemand né à Varsovie et correspondant de la presse allemande depuis vingt-cinq ans, se défend de contribuer au débat sur le « judéo-communisme $»^{30}$, il n'hésite pas à lancer des piques sur le passé de Marcel Reich-Ranicki. Il commente ainsi son entrée dans le Judenrat: « La collaboration ne sembla pas à Reich, alors âgé de 19 ans, être la pire des solutions $»^{31}$. Surtout, il lui reproche de ne pas avoir fait face à son passé dans l'écriture de son autobiographie, en particulier sur son engagement communiste : « La stratégie est claire : minimiser son implication, revêtir l'habit de la victime, et lorsque ce n'est pas la peine car il fut vraiment une victime, adopter la pose du héros $»^{32}$.

Que dit Reich-Ranicki dans son autobiographie au sujet de ses années d'après-guerre en Pologne et pourquoi - et que rajoute Gnauck après son enquête dans les archives polonaises avec quel objectif?

Reich-Ranicki ne cache pas son appartenance au Parti ni ses activités dans le service de censure, à Lublin, Katowice puis Varsovie, avant bientôt d'être envoyé à l'étranger, plus précisément à Berlin puis à Londres, et d'y travailler pour les services de renseignements. Cette proposition qui lui est faite n'est guère surprenante, étant données sa connaissance de l'allemand et ses origines juives, qui font supposer à sa hiérarchie qu'il sera un fidèle collaborateur. Comme lui, de nombreux Juifs polonais sont engagés pour travailler pour le nouveau régime. Ses nouvelles fonctions le conduisent également à opter pour le patronyme à consonance polonaise Ranicki.

30. - Gerhard Gnauck, Marcel Reich-Ranicki..., op. cit., p. 264 : « À ceux qui voudraient considérer mon livre comme une contribution au phénomène du "judéocommunisme", je voudrais dire que je ne prends pas part à ce débat $»$.

31. - Ibid., p. 46.

32. — Ibid., p. 249. 
Sur son bref séjour de deux mois à peine en Silésie, Reich-Ranicki n'est guère disert, si ce n'est pour dire que son travail d'organisateur fut apprécié par ses supérieurs ${ }^{33}$. C'était pourtant l'époque où les Silésiens germanophones et les autres Allemands de la région furent expulsés et remplacés par des Polonais rapatriés des confins orientaux du pays, le tout dans un climat de grande violence et de règlement de comptes. Cette discrétion explique sans doute les insinuations à l'égard de ReichRanicki, accusé d'avoir été complice de ces mauvais traitements, alors qu'aucun document de la police politique n'en fait état.

Puis, il est envoyé en janvier 1946 à la mission militaire polonaise à Berlin sans autre mission précise que celle de «l'observation » pour le compte des services secrets. Si Reich-Ranicki privilégie dans ses mémoires le récit de ses soirées au théâtre ou au concert, les dossiers de la police politique regorgent de rapports d'agents dont le pseudonyme n'a pas à ce jour été dévoilé, et qui informent sur le comportement de leurs collègues, afin de s'assurer de leur loyauté et d'écarter ceux qui seraient suspects. Reich-Ranicki fut-il l'un de ces agents ? C'est ce que pense Gerhard Gnauck, assertions contre lesquelles Reich-Ranicki s'insurgea suite à la parution de cet essai biographique sur ses années polonaises. Pourtant, d'autres documents montrent qu'à l'époque de Berlin, Marcel Reich-Ranicki ne fait pas encore partie des effectifs officiels de la police politique, il n'y entre qu'après son retour de Berlin, fin avril $1946^{34}$.

En février 1948, il est envoyé à Londres, promotion que lui valent ses compétences attestées, pour lesquelles il se voit également décoré de la Croix d'or du Mérite ${ }^{35}$. Il est alors officiellement employé au rang de vice-consul, mais ne fait pas mystère dans ses mémoires de son autre mission, celle du renseignement sur l'émigration polonaise, dont la capitale britannique abritait encore le siège du gouvernement polonais en exil depuis la Seconde Guerre mondiale. Ses mémoires semblent indiquer qu'il n'avait pas de contact direct avec les exilés polonais mais se chargeait seulement de la rédaction de rapports à partir des informations recueillies par ses agents. Les enquêteurs zélés qui ont épluché les archives polonaises ont cependant trouvé que Reich-Ranicki

33. - Marcel Reich-Ranicki, Ma Vie..., op. cit., p. 222.

34. - Documents cités dans l'article à charge contre Reich-Ranicki de Piotr Gontarczyk, « Prawdziwy życiorys Marcelego Reicha » [La véritable biographie de Marcel Reich], Biuletyn Instytutu Pamięci Narodowej, n 1-2 (72-73), 2007, p. 133.

35. - AAN [Archives des Actes Nouveaux], Urząd Rady Ministrów [Bureau du Conseil des Ministres, Gabinet Prezesa RM [Cabinet du président], Dział Odznaczeń [Département des décorations], dossier 1/1-1/136, p. 284 : Wniosek Odznaczeniowy o „Medal Zwycięstwa i Wolności 1945 r." [Décision d'attribution de la " médaille de la Victoire et de la Liberté »] n 17912, 18 IX 1947. Document cité dans Paweł Libera, « Marcel Reich-Ranicki... », art. cit., p. 190, note 39. 
aurait, en vain, tenté de convaincre le journaliste et écrivain Stanisław Cat-Mackiewicz de revenir en Pologne populaire 36.

Enfin, les raisons mêmes avancées par Reich-Ranicki pour son éviction des services secrets l'année suivante en 1949 - le contexte des purges « anti-cosmopolites » en URSS et en Europe centrale, avec leurs accents antisémites, notamment le procès Rajk à Budapest - sont également remises en cause par ces détracteurs de la mémoire. Dans son article consacré à la « véritable biographie de Marcel Reich », l'historien de l'Institut de la Mémoire nationale (IPN), Piotr Gontarczyk, prend au mot les rapports alors rédigés sur le vice-consul à Londres, accusant ce dernier non seulement de «mégalomanie », mais aussi d'avoir falsifié sa biographie en affirmant à tort avoir fait partie du parti communiste polonais avant la guerre. Lesdits rapports reviennent également sur le passé de Reich pendant la guerre et son rôle dans le Conseil juif de Varsovie, ce qui est alors extrêmement mal vu et synonyme de collaboration avec l'ennemi. Enfin, on lui reproche d'entretenir des rapports étroits avec son beau-frère à qui il aurait accordé un visa sans en informer son service et en empochant un pot-de-vin au passage ${ }^{37}$.

Autant d'éléments qui, de façon certaine, ont été utilisés contre Reich pour le renvoyer des services secrets. Mais il est évident qu'il ne s'agissait que de prétextes dans le contexte plus large des purges massives alors menées contre plusieurs centaines d'employés des services du renseignement, le plus souvent d'origine juive, à l'instar du chef du département, le général Komar, qui tombe en mai $1950^{38}$. Dès lors que l'on cherche à se débarrasser d'employés d'origine juive devenus gênants pour le régime, aussi loyaux et fidèles soient-ils, il se trouve aisément des motifs : un rapport négatif sur son comportement, une accusation d'avoir falsifié son autobiographie - pratique alors très répandue visant à faciliter son entrée dans la carrière, ou tout élément disqualifiant idéologiquement, comme son passé au Judenrat ou ses contacts avec des émigrés, membres de sa famille de surcroît.

Reich-Ranicki ne sera pas soumis à procès. Du reste, peu d'hommes et de femmes renvoyés comme lui à la même période subiront des

36. - Gerhard Gnauck, Marcel Reich-Ranicki..., op. cit., p. 150-151 ; Krzysztof Tarka, « "Polska jest Polską”. Powrót Stanisława Mackiewicza do kraju w czerwcu 1956 roku » ['La Pologne c'est la Pologne'. Le retour de Stanisław Mackiewicz au pays en juin 1956], Zeszyty Historyczne [Cahiers historiques] n 150, 2004, p. 55-96 ; Sławomir Cenckiewicz, Piotr Gontarczyk, « Na granicy zdrady. Stanisława Mackiewicza powroty do Polski » [Aux marges de la trahison. Les retours de Stanisław Mackiewicz en Pologne], Biuletyn IPN, n 3-4, 2006, p. 108-109.

37. - Dossier de Marcel Reich : AIPN (Archives de l'Institut de la Mémoire nationale), 0193/896, cité par Piotr Gontarczyk, « Prawdziwy życiorys... », op. cit., p. 129.

38. - Sur ce contexte plus large, voir Audrey Kichelewski, Les survivants..., op. cit., p. 108-124. 
procès, à la différence de ce qui se passait dans les pays voisins. Il passe néanmoins quelques semaines en prison, qu'il relate dans ses mémoires comme un temps pour la lecture et le point de départ d'une nouvelle carrière - avec son talent pour donner une dimension téléologique à sa trajectoire ${ }^{39}$. En effet, à sa sortie de prison, alors même qu'il est exclu du Parti, il est envoyé dans la maison d'édition du ministère de la Défense où il lance une collection portant sur la littérature allemande, évidemment est-allemande. Toutefois, lorsqu'il accepte le poste similaire que lui propose la maison Czytelnik, plus prestigieuse, ce changement lui est interdit et il se retrouve sans travail. Il se met à rédiger des articles et des recensions sur la littérature allemande pour divers journaux polonais. Espérant échapper ainsi aux interdictions de publication, il tente en vain de demander sa réintégration dans le Parti - ce que corroborent ses mémoires comme les archives du Parti ${ }^{40}$. En dépit de son appartenance à l'Union des Écrivains, ses chroniques finissent par tomber sous le coup de la censure. On lui reproche une trop grande proximité intellectuelle avec la culture bourgeoise allemande. Il trouve un nouveau moyen de subsistance en travaillant à la radio polonaise pour la section émettant en allemand. L'interdiction de publication se prolongea jusqu'à la déstalinisation et le changement de contexte culturel. Après 1954, il put à nouveau écrire - un livre sur la littérature allemande paraît l'année suivante et en 1956, avec le Dégel, il est même réintégré dans le Parti. 1956, c'est aussi le moment où il redevient possible de voyager, et même d'émigrer, en tout cas pour de nombreux citoyens d'origine juive qui profitent de cette opportunité pour quitter une Pologne dans laquelle l'antisémitisme refait ouvertement surface ${ }^{41}$. C'est notamment le cas de son cousin Alfred Kalmanowicz, qui part pour Israël avec son épouse au début de l'année 1957. Son dossier personnel fait mention de lettres anonymes reçues sur son lieu de travail - il est alors médecin dans la clinique du ministère des Affaires intérieures ${ }^{42}$.

Marcel et son épouse quittent la Pologne peu après. Dans ses mémoires, il n'évoque pas son cousin mais parle du climat d'antisémitisme de l'époque et surtout, de son désir d'Allemagne qui se cristallise après l'opportunité qu'il a de visiter la RFA en décembre 1957. Il décide alors de fuir en prétextant un voyage d'études dont il ne revient pas, à l'été 1958, tandis que, pour ne pas attirer l'attention, son épouse et leur fils rendent visite à la famille installée en Grande-Bretagne. Ils se retrouvent à Francfort. Marcel ne demande pas l'asile politique, ne

39. - Marcel Reich-Ranicki, Ma Vie..., op. cit., p. 236.

40. — Ibid., p. 243 et Paweł Libera, « Marcel Reich-Ranicki... », art. cit., p. 199-202.

41. - Sur cet épisode, voir Audrey Kichelewski, Les survivants..., op. cit., chapitre 4 et Paweł Machcewicz, « Antisemitism in Poland in 1956 », Polin, n 9, 1994, p. 170-183.

42. — Gerhard Gnauck, Marcel Reich-Ranicki..., op. cit., p. 210-211. 
souhaitant pas être dans l'obligation d'écrire des textes à charge contre la Pologne populaire. Il se spécialise rapidement dans la littérature allemande, sa passion de toujours, délaissant rapidement la littérature polonaise, qu'il commenta encore durant les premières années de son arrivée en RFA. Mais il ne reviendra jamais en Pologne populaire.

\section{Conclusion}

En Pologne, l'intérêt pour Reich-Ranicki s'est éveillé dès les années 1990 suite aux révélations du journaliste polonais Janusz Tycner dans Die Zeit en 199443, utilisant les archives récemment ouvertes du Parti, et s'est encore accru suite à la parution du livre de Gnauck. De nombreux journalistes et quelques chercheurs se sont alors plongés dans les documents d'archives, plus encore après la publication de son autobiographie par Marcel Reich-Ranicki. Il s'agissait certes de faire connaître le passé polonais de ce critique littéraire si célèbre, mais aussi et surtout de montrer sa part d'ombre, voire de ternir son image. En soulignant ses services rendus au régime communiste ou encore son rôle dans le Conseil juif, il s'agissait bien de le faire passer pour un traître à toutes les causes et à toutes ses « patries ». Pour autant, tous les éléments soidisant « dévoilés » par Gnauck, ses prédécesseurs et ses successeurs sur le passé communiste et " d'espion » de Reich-Ranicki figuraient en réalité déjà dans son autobiographie, rédigée après ces premières « révélations » et ne cherchant aucunement à les nier. Reich-Ranicki ne cachait pas avoir appartenu au Parti, avoir travaillé pour les services secrets, avouant même que « c'était sans doute [là] une grave erreur » ${ }^{44}$. Comme l'a bien remarqué la chercheuse Joanna Tokarska-Bakir ${ }^{45}$, ces écrits ne visent qu'à salir la mémoire de l'homme et n'apportent en réalité aucun éclairage particulier ou nouveau, même lorsqu'ils abondent en citations de dossiers des services secrets. D'autant plus que ces dossiers sont à présent consultables aux archives de l'Institut de la Mémoire nationale, dont l'une des missions est précisément de débusquer le passé trouble de citoyens occupant une place importante dans la vie publique ${ }^{46}$, en

43. - Janusz Tycner, « Die Akte Ranicki », Die Zeit, 15.07. 1994, p. 39-40.

44. - Marcel Reich-Ranicki, Ma Vie..., op. cit., p. 231.

45. - Joanna Tokarska-Bakir, " Unbearable whispering », Biweekly. Link with culture, $\mathrm{n}^{\circ} 2$, mai 2010, traduit du polonais par Michał Czarniecki, accessible à l'adresse suivante : https://www.biweekly.pl/article/1188-unbearable-whispering.html?print=1 (consulté le 24/7/2019).

46. - Sur l'Institut de la Mémoire nationale, voir notamment Valentin Behr, « Le ministère de la mémoire », La Vie des idées, 11/04/2014 : https://laviedesidees. fr/Le-ministere-de-la-memoire.html (consulté le 24/7/2019) et Georges Mink, « Les usages des Instituts de la mémoire nationale (IMN) dans les recompositions partisanes (1989-2008) », in : Pascal Bonnard, Georges Mink (dir.), Le passé au présent, Paris, Michel Houdiard, 2011, p. 171-184. 
Pologne, mais aussi au-delà des frontières du pays, comme le montre le cas de Reich-Ranicki. La place importante consacrée à la judéité de ce dernier dans son lien avec le régime communiste est également un sous-entendu constant de ce type de publication, entretenant le mythe d'un prétendu judéo-bolchevisme ayant nui aux intérêts de la " vraie » Pologne, catholique et national(ist)e.

L'ultime chapitre que Gnauck consacre aux années polonaises de Reich-Ranicki s'intitule précisément «Biographie ou jeu » et fait le point sur les imprécisions et le manque de transparence imputables à ce dernier, reproche que reprendront à leur compte un certain nombre d'auteurs après lui, de manière parfois plus virulente encore. Tous citent alors une phrase que Reich-Ranicki se défend d'avoir jamais prononcée : « Je suis à moitié Polonais, à moitié Allemand et totalement Juif », afin de souligner dans un sous-entendu complice avec leurs lecteurs que l'identité juive subsumerait les autres identités nationales et fournirait une clef explicative à ses trahisons successives. Ces auteurs ont assurément propagé en Pologne et au-delà une image plus trouble du grand critique littéraire, qui n'est plus là pour se défendre lorsque ses vidéos sont commentées en 2019 par des propos haineux. Des propos dont il est permis de penser qu'ils ont été en partie inspirés par ce flot d'écrits très critiques sur les années polonaises de Marcel Reich-Ranicki. Il serait pourtant bien plus correct de dire de ce dernier qu'il était tout autant juif, polonais et allemand. 